\title{
Amélioration de la traçabilité des mesures environnementales de radioactivité via la production de matériaux marqués
}

\section{Preparation of spiked matrices to improve the traceability of environmental radioactivity measurements}

\author{
Valérie LOURENÇO, Didier LACOUR, Laurent FERREUX, Isabelle LE GARRÉRÈS et Sophie MORELLI
}

CEA, LIST, Laboratoire national Henri Becquerel (LNE-LNHB), 91191 Gif-sur-Yvette Cedex, France, valerie.lourenco@cea.fr.

\section{Résumé}

La surveillance de la radioactivité dans l'environnement est l'affaire de tous, et assurer la traçabilité des mesures réalisées est essentiel. Aussi, si la réglementation française impose aux exploitants d'installations nucléaires leurs propres dispositifs de contrôle de la radioactivité, ces laboratoires doivent être agréés par l'Autorité de Sûreté Nucléaire (ASN). Cet agrément est accordé pour une durée de trois à cinq ans sur la base de résultats de tests réglementaires élaborés par l'Institut de Radioprotection et de Sûreté Nucléaire (IRSN). Le Laboratoire national Henri Becquerel (LNE-LNHB), en tant que laboratoire national de métrologie pour les rayonnements ionisants, a été sollicité pour établir des références nationales dans le domaine des mesures d'activité pour l'environnement. Un programme de recherche lancé en 2009 au LNE-LNHB avait pour but de produire des matériaux de référence traçables, de masse volumique et de composition proches des échantillons usuellement prélevés dans l'environnement, afin de permettre aux laboratoires d'étalonner leurs instruments de mesure et de se préparer aux tests interlaboratoires de l'IRSN. Dans le cadre de ce programme, le LNE-LNHB a mis au point une méthode de marquage fiable avec un rendement proche de $100 \%$ permettant à la fois de réaliser des matrices de masse volumique et de composition en radionucléides variées et de limiter la durée des mesures. In fine, le marquage est réalisé en milieu humide, sous agitation, dans un solvant de faible température d'ébullition au moyen d'un évaporateur rotatif. Cette méthode a été testée avec succès pour l'élaboration de matrices solides à base d'herbe, de chou et sur une base de terre végétale. L'article décrit la mise au point du protocole de préparation de matrices marquées avec des radionucléides émetteurs gamma ainsi que la caractérisation des matrices marquées. L'une de ces matrices a fait l'objet d'un test interlaboratoire dont les résultats sont abordés dans l'article.

MOTS CLÉS : TRAÇABILITÉ, MATRICE MARQUÉE, ÉMETTEUR GAMMA, MATÉRIAU DE RÉFÉRENCE, RADIOACTIVITÉ ENVIRONNEMENTALE, TEST INTERLABORATOIRE.

\begin{abstract}
The monitoring of environmental radioactivity is important for public health protection. In France, environmental radioactivity is specifically monitored by a network of certified laboratories. Indeed the Nuclear Safety Authority (ASN) delivers three to five year governmental agreements to each laboratory provided that it succeeds in proficiency tests (PTs) organized by the Institute for Radiological Protection and Nuclear Safety (IRSN). To ensure a direct traceability chain in radioactivity measurements, the Laboratoire National Henri Becquerel (LNE$L N H B$ ), as the French national laboratory for radionuclide metrology, has been organizing national PTs for more than 40 years. LNE-LNHB also regularly realizes specific PTs to train the laboratories to the regulatory tests of IRSN. Most tests are based on aqueous solutions but there is a growing demand for tests on solid matrices to be measured by $\gamma$-spectrometry. Measurement of radionuclides from environmental samples includes a wide variety of matrix compositions and densities. Since 2009, LNE-LNHB is working on the production of suitable calibration reference materials to improve the traceability of environmental radioactivity measurements in France. To address this issue, LNE$L N H B$ intends to produce mixed $\gamma$-ray reference materials with a known mass activity and a composition as representative as possible of real environmental samples. The use of such materials will also improve the calibration of $\gamma$-spectrometry measurement systems due to a more accurate determination of the self-attenuation correction by measuring a known sample whose composition is close to the real one. This paper describes the development of the preparation protocol and the characterization of traceable matrices, spiked with various $\gamma$-ray emitters. A $P T$ exercise has been organized with a low density matrix produced. The results of the participants are mentioned in this article.
\end{abstract}

KEY WORDS: SPIKED MATRICE, MIXED GAMMA EMITTER REFERENCE MATERIAL, ENVIRONMENTAL RADIOACTIVITY, PROFICIENCY TEST. 


\section{La surveillance de l'environnement}

L'état radiologique de l'environnement et l'impact des installations nucléaires sur ce dernier sont surveillés par le Réseau National de Mesures (RNM) de la radioactivité de l'environnement, piloté par l'Autorité de Sûreté Nucléaire (ASN) et son expert technique l'Institut de Radioprotection et de Sûreté Nucléaire (IRSN). Le RNM a été créé dans le but de répondre aux exigences réglementaires mentionnées dans le code de la santé publique (article R 1333-11) et dans le décret $\mathrm{N}^{\circ}$ 2007-1582 du 7 novembre 2007. Les analyses radiologiques des échantillons environnementaux sont réalisées dans ce cadre par l'IRSN et les laboratoires agréés. Les résultats des mesures sont, depuis 2010, mis en ligne sur un site internet dédié (http://www.mesure-radioactivite.fr/), consultable par tous.

\subsection{Les tests réglementaires}

Les agréments sont accordés sur la base de critères qualité (notamment la conformité des pratiques avec les exigences NF EN ISO/CEI 17025) et de critères techniques, à savoir l'obtention de résultats satisfaisants aux tests interlaboratoires pluriannuels organisés par l'IRSN. Les différents essais interlaboratoires sont programmés par type de matrice environnementale et par catégorie de mesures radioactives en fonction de la grille d'agrément homologuée par l'ASN. Il existe ainsi six familles de matrices environnementales (eaux, sols, matrices biologiques, etc.) et dix-sept catégories de mesures radioactives (tritium, alpha global, bêta global, radionucléides émetteurs $\gamma$ d'énergie supérieure à $100 \mathrm{keV}$, etc.), représentant 52 agréments. Les résultats des tests sont analysés par une commission d'agrément. Un écart supérieur à $25 \%$ par rapport aux valeurs de référence entraîne le retrait de l'agrément. Dans ce cas, le laboratoire ne peut plus réaliser de mesures en rapport avec l'agrément perdu.

Compte tenu de cet enjeu, certains laboratoires participants ont sollicité le LNE-LNHB en tant que laboratoire national de métrologie et pour son expérience dans l'organisation de tests interlaboratoires depuis une quarantaine d'années, afin d'organiser des tests de préparation aux tests réglementaires de l'IRSN.

Sous la pression de la réglementation, le besoin de traçabilité métrologique dans le domaine des mesures d'activité pour l'environnement augmente. Ce besoin n'est actuellement couvert que partiellement par le LNELNHB via l'organisation de tests interlaboratoires pour les faibles valeurs d'activité sur des échantillons traçables liquides.

\subsection{La traçabilité des mesures environnementales}

Dans ce domaine, les laboratoires sont raccordés aux étalons nationaux de manière très indirecte :

- en réalisant eux-mêmes leurs étalons par incorporation de solutions étalons dans leurs matrices d'intérêt;
- en raccordant leurs appareils de mesure avec des eìtalons du commerce, qui sont peu repreìsentatifs des matrices environnementales (solutions aqueuses acides de masse volumique voisine de $1 \mathrm{~kg} \cdot \mathrm{L}^{-1}$ );

- en utilisant des matériaux de référence (certifiés ou non) pour l'étalonnage des appareils de mesure. Ces matériaux sont soit des matériaux réels ayant subi une contamination dont le contenu radioactif est caractérisé [1-3], soit des matrices simplifiées marquées avec une quantité connue de radioactivité $[4,5]$.

La traçabilité de ces matériaux réels est assurée, puisque les valeurs de référence sont mesurées par des laboratoires nationaux de métrologie, mais la composition et la masse volumique des matériaux sont imposées par la nature de la matrice prélevée. Cependant, l'utilisation de matrices réelles caractérisées est restreinte. En effet, la réalisation de ces matrices (homogénéisation, mesures, etc.) est très coûteuse du fait de la longue durée des mesures. Ces matériaux sont donc peu nombreux et, de ce fait, bien connus des utilisateurs. Leur utilisation dans le cadre de tests interlaboratoires est limitée puisque ces matériaux de composition fixe sont ensuite facilement identifiables.

Pour les laboratoires accrédités, la participation aux essais interlaboratoires ainsi que l'utilisation de matériaux de référence certifiés (MRC) est vivement recommandée dans les normes comme la norme NF EN ISO/CEI 17025 [6], pour démontrer leurs aptitudes de mesure. Si la base de données COMAR (COde d'indexation des MAtériaux de Référence) [7], initiée par le LNE dans les années 1970, recense environ 11000 matériaux de référence, il est important de noter que ceuxci répondent surtout aux besoins de traçabilité des analyses chimiques et biologiques. En effet, les matériaux caractérisés en termes de radioactivité ou de composition isotopique ne représentent qu'entre $1 \%$ et $2 \%$ de ces matériaux. Au total, on ne dénombre qu'une trentaine de matériaux de référence utilisables dans le domaine de la radioactivité environnementale et le stock de certains arrive à épuisement.

Les travaux du LNE-LNHB dans le domaine de la production de matériaux de référence par marquage ainsi que leur utilisation dans les tests interlaboratoires répondent aux besoins de traçabilité des mesures environnementales de radioactivité en France.

\subsection{Fabrication de matériaux de référence par marquage}

Face à la diversité des échantillons environnementaux, en particulier solides, la mise au point d'une méthode de marquage fiable permettrait d'une part de fabriquer des matrices de masse volumique et composition en radionucléides variées, et d'autre part de limiter la durée des mesures par le recours à une méthode de marquage dont le rendement serait caractérisé (et idéalement voisin de $100 \%)$.

Le LNE-LNHB souhaitait produire divers matériaux de référence traçables, de masse volumique et de 
composition proches des échantillons usuellement prélevés pour le suivi de la radioactivité environnementale, afin de permettre aux laboratoires d'étalonner leur instrumentation ou de tester leur capacité de mesure par le biais de tests interlaboratoires.

L'article décrit la mise au point de la préparation et la caractérisation de matrices marquées avec des radionucléides émetteurs gamma. Afin d'établir le rendement de la méthode de marquage, les valeurs de référence obtenues par mesure des échantillons ont été comparées avec la quantité de radioactivité ajoutée lors du marquage. L'une des matrices produites a fait l'objet d'un essai interlaboratoire dont les résultats sont présentés.

\section{Préparation de matrices environnementales traçables}

Les activités massiques des échantillons environnementaux étant très faibles, de l'ordre de quelques becquerels par kilogramme, il est impératif d'éviter toute contamination lors des étapes de préparation et de mesure. Pour cela, le LNE-LNHB a procédé en 2009 à l'aménagement de locaux réservés à la préparation des matrices, physiquement séparés des locaux dédiés aux opérations de radiochimie de « haute » valeur d'activité, et équipés de matériels dédiés eux-aussi aux faibles valeurs de radioactivité (ventilation, appareils de broyage selon la dureté des matrices, tamiseuses, etc.).

\section{1. Étapes de préparation d'une matrice traçable}

Les matrices sont destinées à être mesurées par spectrométrie gamma, ce qui implique qu'elles devront être homogènes en termes de masse volumique (autoabsorption) et d'activité massique (absence de points chauds). Pour cela, il est nécessaire de diviser finement le matériau retenu, un tamisage autour de $250 \mu \mathrm{m}$ étant le plus communément choisi pour les matrices solides de type sols par exemple.

Les différentes étapes pour obtenir une matrice marquée satisfaisante, sont les suivantes :

- E1. Choix d'un échantillon homogène d'une matrice, dont il convient de mesurer le bruit de fond radioactif puisque qu'il s'agit d'une matrice naturelle.

- E2. Séchage afin de faciliter l'étape de broyage et d'augmenter la durée de conservation de la matrice en diminuant sa teneur en eau. Cette étape est réalisée au moyen d'une étuve à convection forcée à $60^{\circ} \mathrm{C}$. En général, après $48 \mathrm{~h}$, la masse du matériau n'évolue plus.

- E3. Broyage afin d'obtenir une matrice finement divisée. Pour les matériaux fibreux, cette étape est réalisée à l'aide d'un broyeur à couteaux.

- E4. Tamisage afin de caractériser la distribution granulométrique des particules et de récupérer la fraction granulométrique voulue.
- E5. Homogénéisation par mélange de tous les lots traités et évaluation de la masse volumique et du taux d'humidité de la matrice traitée.

- E6. Marquage de la matrice au moyen d'une méthode permettant une répartition homogène de la radioactivité.

- E7. Mesures par spectrométrie gamma afin de caractériser la matrice marquée obtenue en termes d'activités massiques moyennes et d'en vérifier l'homogénéité à l'échelle de sous-lots (de taille inférieure au conditionnement final pour vérifier l'absence de points chauds).

\subsection{Choix des matrices brutes}

\subsubsection{Critères de choix}

Dans le but de préparer des matrices naturelles marquées artificiellement, des essais préliminaires ont été réalisés. Il s'agit d'abord de vérifier le comportement de différents matériaux lors du traitement nécessaire pour conduire à un marquage.

La fourniture de matrices marquées offre l'avantage de travailler sur des matériaux dont les masses volumiques ont des valeurs pouvant être comprises entre $0,15 \mathrm{~kg} \cdot \mathrm{L}^{-1}$ pour les plus légers tels que la sciure et $1,3 \mathrm{~kg} \cdot \mathrm{L}^{-1}$, par exemple pour des terres végétales.

Les critères de sélection de la matrice sont entre autres :

- la masse volumique : d'après une enquête réalisée auprès des laboratoires de mesures de matrices environnementales, une masse volumique voisine de $0,5 \mathrm{~kg} \cdot \mathrm{L}^{-1}$ est la valeur la plus utilisée. Toutefois, des valeurs inférieures peuvent être acceptables si le produit reste manipulable y compris après l'étape de marquage;

- la distribution granulométrique : pour des raisons de manipulation après marquage, il est nécessaire d'avoir une faible proportion de très petites particules (en général inférieures à environ $100 \mu \mathrm{m})$. En effet, l'expérience montre que, plus il y a de particules fines, plus le matériau est électrostatique et difficile à manipuler. De plus, une importante quantité de particules fines en présence de particules plus grosses conduit à un tassement du matériau traité après conditionnement. Cette variation de la masse volumique au sein du volume de mesure perturbe les mesures en spectrométrie $\gamma$;

- le taux d'humidité : le matériau doit présenter un taux d'humidité maximal de quelques unités pourcent. En effet, cela permet d'éviter qu'il ait tendance à s'agglomérer une fois conditionné, ce qui conduirait à la formation de points de densité plus élevée. Par ailleurs, une teneur faible en eau permet d'améliorer sa stabilité dans le temps en limitant les processus de dégradation dans le cas des matrices d'origine végétale. Il est de plus nécessaire de connaître le taux d'humidité de telles matrices car les résultats des mesures 
Tableau 1

Récapitulatif des masses volumiques $\left(\mathrm{kg} \cdot \mathrm{L}^{-1}\right)$ obtenues après broyage et prétamisage à $1 \mathrm{~mm}$ ou à $250 \mu \mathrm{m}$ (repéré par *).

\begin{tabular}{|c|c|c|c|c|}
\hline Matrice & Herbe* $^{*}$ & $\begin{array}{c}\text { Copeaux } \\
\text { de bois }\end{array}$ & Lentilles & Pâtes \\
\hline $\begin{array}{c}\text { Masse } \\
\text { volumique }\end{array}$ & 0,37 & 0,15 & 0,75 & 0,84 \\
\hline Matrice & $\mathbf{R i z}$ & $\begin{array}{c}\text { Café en } \\
\text { grains }\end{array}$ & $\begin{array}{c}\text { Brisures } \\
\text { de thé }\end{array}$ & $\begin{array}{c}\text { Terre } \\
\text { végétale* }\end{array}$ \\
\hline $\begin{array}{c}\text { Masse } \\
\text { volumique }\end{array}$ & 0,78 & 0,37 & 0,43 & 1,20 \\
\hline
\end{tabular}

de radioactivité doivent être donnés en becquerels par gramme ou kilogramme de matériau sec ;

- le coût : celui-ci doit rester raisonnable car de grandes quantités initiales de matériau sont parfois nécessaires puisqu'il arrive que seul le dixième de la masse de matrice initiale se retrouve dans le matériau marqué.

\subsubsection{Tests de huit matrices : broyage et distribution granulométrique}

Des essais ont été réalisés sur huit matrices afin de faire une sélection selon leur masse volumique après broyage et leur représentativité environnementale. Les matrices testées sont : l'herbe, les copeaux de bois, les lentilles, les pâtes, le riz, le café en grains, les brisures de thé et la terre végétale.

Si nécessaire, les matériaux sont séchés avant broyage. C'est le cas de la matrice « herbe» qui contient une proportion d'eau particulièrement importante $(90 \%)$. Un broyeur à couteaux a été utilisé pour le broyage de tous les matériaux sauf la terre végétale, pour laquelle un broyeur à jarres était plus indiqué.

Les résultats obtenus sont regroupés dans le tableau 1.

L'étape de broyage s'est avérée difficile dans le cas des copeaux de bois, la sciure obtenue étant apparemment constituée d'une proportion importante de particules fines. Les grains de riz et de café ont colmaté le broyeur malgré le prétamisage à $1 \mathrm{~mm}$ au lieu de $250 \mu \mathrm{m}$ habituellement.

L'étude de la distribution granulométrique s'est faite sur des fractions de $20 \mathrm{~g}$ de produits séchés, broyés et prétamisés à $1 \mathrm{~mm}$. Pour chaque matrice, en plus de la gamelle (récipient qui recueille les particules les plus petites), la même série de tamis est utilisée $(0,25 \mathrm{~mm}$, $0,18 \mathrm{~mm}, 0,15 \mathrm{~mm}, 0,106 \mathrm{~mm}$ ). La fréquence de vibration est réglée sur $50 \mathrm{~Hz}$ et l'opération s'est déroulée en deux séquences de 10 min. Les tamis sont pesés vides au début de l'opération et pleins après la fin de chaque séquence.

Après les essais, les tamis sont nettoyés méticuleusement au pinceau et à la soufflette avec de l'air comprimé en se plaçant sous l'appareil de filtration d'air car l'opération de nettoyage génère une quantité importante de poussière.
Tableau 2

Distribution granulométrique (en \% de masse totale).

\begin{tabular}{|c|c|c|c|c|c|}
\hline $\begin{array}{l}\text { Taille des } \\
\text { particules }\end{array}$ & $<106 \mu \mathrm{m}$ & $\begin{array}{l}<150 \mu \mathrm{m} \\
>106 \mu \mathrm{m}\end{array}$ & \begin{tabular}{|l|l|}
$<180 \mu \mathrm{m}$ \\
$>150 \mu \mathrm{m}$
\end{tabular} & \begin{tabular}{|l|}
$<250 \mu \mathrm{m}$ \\
$>180 \mu \mathrm{m}$ \\
\end{tabular} & $>250 \mu \mathrm{m}$ \\
\hline Herbe & 47 & 25 & 19 & 8 & 0 \\
\hline $\begin{array}{c}\text { Copeaux } \\
\text { de bois }\end{array}$ & 14 & 10 & 14 & 22 & 40 \\
\hline Lentilles & 2 & 3 & 6 & 14 & 75 \\
\hline Pâtes & 3 & 3 & 2 & 4 & 88 \\
\hline Riz & $<1$ & $<1$ & 1 & 5 & 93 \\
\hline $\begin{array}{c}\text { Café } \\
\text { en grains }\end{array}$ & $<1$ & $<1$ & $<1$ & 6 & 93 \\
\hline $\begin{array}{c}\text { Brisures } \\
\text { de thé }\end{array}$ & \multicolumn{4}{|c|}{6} & 94 \\
\hline $\begin{array}{c}\text { Terre } \\
\text { végétale }\end{array}$ & \multicolumn{4}{|c|}{97} & 3 \\
\hline
\end{tabular}

Les résultats obtenus sont présentés dans le tableau 2. La distribution granulométrique selon la taille de particules est donnée en pourcentage de masse d'une classe de tailles par rapport à la masse totale. D'un lot à un autre, la variabilité des valeurs est de l'ordre de $3 \%$.

La sciure et l'herbe broyée sont les matrices qui ont présenté le caractère hygroscopique le plus important rendant leur manipulation délicate. Une fois broyés, de nombreux matériaux deviennent électrostatiques. C'est le cas en particulier de l'herbe, des copeaux de bois et du café ainsi que, dans une moindre mesure, du riz et des pâtes.

Enfin, la facilité de manipulation d'une matrice lors de l'ensemble des étapes semble être corrélée à la présence de particules fines $(<106 \mu \mathrm{m})$ car les matrices les plus délicates à travailler se sont révélées être l'herbe broyée et la sciure.

\subsection{Mise au point de la procédure de marquage}

\subsubsection{Choix de la matrice et valeur de marquage radioactif}

Pour conduire ces essais, la matrice herbe broyée a été retenue pour sa représentativité environnementale. En effet, des prélèvements d'herbe sont régulièrement réalisés par les laboratoires chargés du suivi de la radioactivité environnementale.

Avant de procéder à son marquage, la matrice brute a été mesurée afin de quantifier son activité avant dopage. Ces mesures ont été réalisées sur la matrice broyée après tamisage à $250 \mu \mathrm{m}$.

Parmi les radionucléides artificiels, seul le ${ }^{137} \mathrm{Cs}$ est présent avec une activité massique de $(2,5 \pm 0,2) \mathrm{Bq} \cdot \mathrm{kg}^{-1}$ (de matière sèche). Cette matrice est donc tout à fait convenable pour l'étude de marquage. Idéalement, afin de tester l'aptitude des laboratoires à contrôler la radioactivité environnementale, la valeur adéquate de marquage doit être entre deux et trois fois supérieure à l'activité du matériau «blanc ». Néanmoins, dans le cadre de cette étude de faisabilité, la valeur de marquage retenue est 


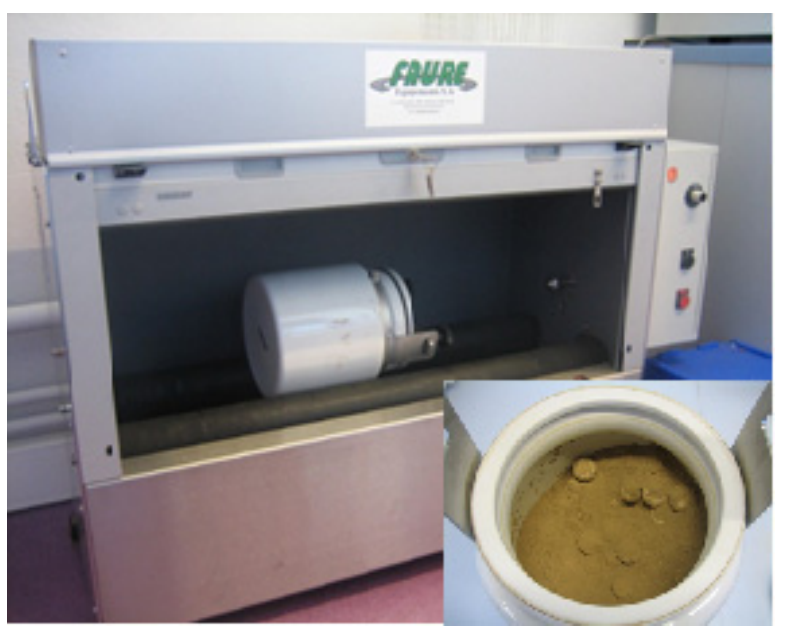

Fig. 1. - Broyeur à jarres présenté avec une jarre en place et vue d'une jarre ouverte après une séquence de broyage/ homogénéisation.

de $100 \mathrm{~Bq} \cdot \mathrm{kg}^{-1}$ à $1000 \mathrm{~Bq} \cdot \mathrm{kg}^{-1}$ (de matière sèche) afin de limiter les temps de comptage à quelques jours.

\subsubsection{Méthodes de marquage possibles}

L'objectif de production de matrices marquées sousentend de réaliser des volumes importants de matrices homogènes. Par exemple, lors des tests d'aptitude organisés par le LNE-LNHB sur des solutions aqueuses, le nombre moyen de participants est de l'ordre de vingt. Pour les faibles valeurs de radioactivité, il est nécessaire d'envoyer environ $0,5 \mathrm{~L}$ de matrice à chaque participant. Ainsi, il apparaît que le procédé à retenir doit permettre la préparation d'environ $20 \mathrm{~L}$ de matrice marquée.

Compte tenu des appareils disponibles au laboratoire, plusieurs stratégies de marquage sont envisageables :

- marquage à sec puis homogénéisation à l'aide d'un agitateur tridimensionnel [8-10];

- marquage à l'aide du broyeur à jarres qui permet de travailler en milieu humide ou sec puisqu'il fait également office de moyen d'homogénéisation;

- marquage à l'aide de l'évaporateur rotatif avec un solvant de faible température d'ébullition [11].

Pour les essais de marquage à sec, le flacon final est rempli avec la matrice à marquer, l'activité est ajoutée à l'aide d'une micropipette de $1 \mathrm{~mL}$ et une fois l'ajout séché, le flacon est laissé dans un agitateur tridimensionnel pendant a minima une nuit. Cette méthode ne permet pas l'obtention d'échantillons marqués homogènes même si elle présente l'avantage de fabriquer des échantillons directement dans le flacon final.

L'utilisation du broyeur à jarres, illustré en figure 1, permet de mettre en contact la matrice à marquer, la solution de marquage et des billes de céramique. La rotation de la jarre permet de mettre en contact intime les différentes phases. Toutefois, cette méthode présente des inconvénients : il sera nécessaire de vérifier la

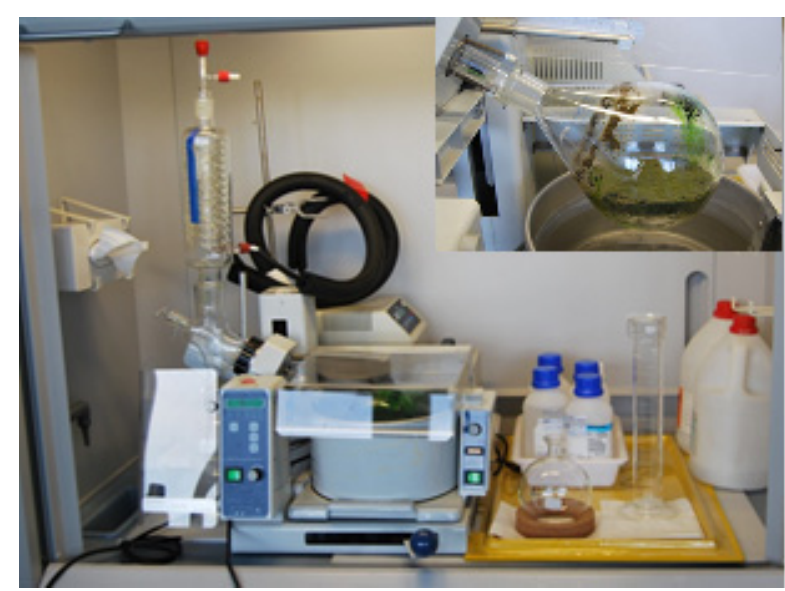

Fig. 2. - Poste de marquage avec l'évaporateur rotatif et vue du contenu d'un ballon après l'évaporation du solvant.

non-contamination de la jarre et surtout des billes de céramique, voire de les décontaminer. En outre, une vérification de la granulométrie par tamisage à l'issue du marquage impliquerait aussi un risque de contamination des jeux de tamis.

L'utilisation des jarres d'un volume d'environ $10 \mathrm{~L}$ permet de marquer environ $3 \mathrm{~L}$ de matière en une fois. Il faut donc plusieurs marquages pour atteindre les $20 \mathrm{~L}$ nécessaires. Enfin, si le marquage est réalisé en milieu humide, l'évaporation du solvant de marquage doit faire l'objet d'une étape additionnelle.

L'utilisation de l'évaporateur rotatif présenté en figure 2 limite la quantité de matière qu'il est possible de marquer en une fois (environ $0,5 \mathrm{~L}$ ). La rotation simple du ballon lors du marquage permet d'homogénéiser le lot marqué. Le marquage a lieu dans un récipient en verre, matériau qui, à $\mathrm{pH}$ acide, ne favorise pas la rétention des radionucléides par adsorption. De plus, la décontamination de surfaces en verre est mieux maîtrisée au laboratoire que celle des surfaces céramiques (plus poreuses).

La rotation du ballon contenant la matrice à marquer et la solution de marquage assure un contact intime entre les deux phases. Le chauffe-ballon et le système de réfrigération permettent ensuite d'évaporer et de récupérer immédiatement le solvant de marquage. Une mesure de l'activité massique du solvant après marquage permet une première évaluation du rendement de marquage de la matrice. Afin de faciliter l'opération de marquage, nous avons choisi de réaliser le marquage dans de l'acétone du fait de sa faible température d'ébullition $\left(56^{\circ} \mathrm{C}\right)$. Le méthanol a été envisagé car ce solvant présente des propriétés physico-chimiques voisines de l'eau (polarité et constante diélectrique notamment). Toutefois, le méthanol n'a pas été retenu, le seuil de toxicité étant facilement atteint bien avant que l'on sente son odeur.

\subsubsection{Essais de marquage}

Pour les essais de marquage, un cocktail d'émetteurs gamma représentatifs du futur mélange de marquage complet est utilisé : ${ }^{137} \mathrm{Cs},{ }^{60} \mathrm{Co}$ et ${ }^{109} \mathrm{Cd}$. 
Le ${ }^{137}$ Cs présente l'avantage d'être mono-énergétique et son émission vers $662 \mathrm{keV}$ ne devrait pas être trop atténuée dans la matrice. Le ${ }^{60}$ Co présente deux émissions de plus haute énergie (énergie moyenne de 1,25 MeV). Enfin, le ${ }^{109} \mathrm{Cd}$, mono-énergétique de basse énergie (vers $88 \mathrm{keV}$ ), est plus difficile à mesurer, mais il permet d'évaluer la maîtrise des corrections d'auto-atténuation dans la matrice.

D'un point de vue chimique, le césium devrait se comporter comme les autres alcalins qui seront ajoutés dans le mélange final ou présents naturellement (Na et K). Le cobalt et le cadmium sont des éléments de transition à la chimie spécifique et pourraient se distribuer de façon différente; il est donc nécessaire de les inclure dans les tests de marquage pour vérifier ce point.

La solution de marquage acide (en milieu $\mathrm{HNO}_{3}$ $1 \mathrm{~mol} \cdot \mathrm{L}^{-1}$ ) est composée des entraîneurs (isotopes stables) des trois éléments cités et contient : ${ }^{109} \mathrm{Cd}$ à environ $125 \mathrm{kBq} \cdot \mathrm{kg}^{-1},{ }^{60} \mathrm{Co}$ et ${ }^{137} \mathrm{Cs}$ autour de $25 \mathrm{kBq} \cdot \mathrm{kg}^{-1}$. L'activité massique de cette solution de marquage simplifiée est mesurée par spectrométrie gamma (géométrie SG500 de 0,5 L).

Lors des essais réalisés avec des valeurs d'activité plus importantes que celles retenues au final pour le marquage, les mesures sur les échantillons marqués une fois secs suggèrent que le rendement du marquage soit proche de $100 \%$ quel que soit le radionucléide considéré. Toutefois, afin de vérifier ce point, des mesures plus longues, toujours par spectrométrie gamma, sont réalisées sur le solvant recondensé dans le réfrigérant et sur les eaux de nettoyage des ballons entre chaque marquage. Ces mesures montrent que la radioactivité reste sur la matrice car les valeurs d'activité mesurées représentent de l'ordre de $0,1 \%$ de l'activité totale utilisée pour les marquages.

\subsubsection{Procédure de marquage retenue}

Une aliquote pesée de $0,5 \mathrm{~L}$ de la matrice broyée est introduite dans un flacon d'évaporation de $2 \mathrm{~L}, 500 \mathrm{~mL}$ d'acétone pour analyse sont ajoutés ainsi qu'un millilitre de la solution de marquage à l'aide d'une micropipette dont la reproductibilité a été caractérisée. Le mélange obtenu a la consistance d'une boue fine. Le flacon est laissé en rotation pendant environ $1 \mathrm{~h}$ afin d'homogénéiser la radioactivité au sein du mélange. La température du bain est alors augmentée vers $60{ }^{\circ} \mathrm{C}$ afin d'évaporer l'acétone qui sera ensuite condensée dans le réfrigérant. Là encore, l'acétone est recueillie en partie et conditionnée pour mesure par spectrométrie gamma pour compléter la détermination des rendements de marquage. Pour produire les $10 \mathrm{~L}$ de matrice marquée, vingt marquages successifs sont nécessaires. Une étape finale d'homogénéisation de l'ensemble des lots est réalisée manuellement par quartages successifs à l'aide de quatre récipients de 10 L. La matrice marquée est ensuite conditionnée dans des flacons opaques (pour la protéger de la lumière) de $0,5 \mathrm{~L}$ correspondant à une géométrie directement mesurable par les participants des essais interlaboratoires du LNE-LNHB. Du fait du fort caractère électrostatique de la matrice marquée, ces opérations sont réalisées en boîte à gants afin d'éviter tout risque de contamination des opérateurs ou des locaux des laboratoires susceptibles de participer au test interlaboratoires.

Le taux d'humidité des échantillons après marquage est déterminé par séchage de plusieurs aliquotes à l'étuve à convection forcée selon la même procédure que l'étape de séchage $\left(60{ }^{\circ} \mathrm{C}\right.$ pendant $\left.48 \mathrm{~h}\right)$. Il en résulte un taux d'humidité de $(2,96 \pm 0,02) \%$.

\subsubsection{Traçabilité des échantillons produits}

Le mélange de marquage utilisé est préparé par dilutions gravimétriques à partir de solutions très actives mesurées par chambre d'ionisation avec des incertitudes types relatives de l'ordre de $0,3 \%$. Les chambres d'ionisation utilisées sont des instruments de mesure secondaires, raccordés aux références primaires du LNELNHB, qui présentent des incertitudes de mesures faibles au regard des incertitudes des participants aux tests interlaboratoires, en particulier pour les faibles valeurs d'activité (entre $5 \%$ et $10 \%$ à $k=1$ ). En complément, afin d'assurer la redondance métrologique, la solution de marquage préparée est mesurée par spectrométrie gamma, autre technique de mesure secondaire du laboratoire, présentant des incertitudes types de quelques unités pourcent.

Le marquage est réalisé en utilisant une micropipette dont la reproductibilité est caractérisée gravimétriquement et qui est égale à $0,2 \%$.

Dans le cas des échantillons liquides produits habituellement, pour lesquels toutes les dilutions se font par pesée et dont l'homogénéité est vérifiée, les valeurs de référence retenues sont celles des mesures réalisées sur les échantillons finaux. Dans le cas des échantillons multigamma, cette mesure se fait par spectrométrie gamma. La compatibilité des valeurs de référence obtenues avec les valeurs issues des mesures en chambre d'ionisation et des coefficients de dilution associés à la préparation est vérifiée à l'incertitude type près.

Dans le cas des matrices marquées, l'étape de marquage présente le risque de ne pas être quantitative. $\mathrm{Ce}$ sont donc les valeurs de référence mesurées par spectrométrie gamma sur les échantillons finaux qui ont été retenues et qui servent d'ailleurs à évaluer les rendements de marquage.

\subsection{Mesures par spectrométrie gamma}

Les mesures par spectrométrie gamma ont été réalisées à l'aide d'un spectromètre gamma bas bruit de fond en germanium hyper-pur, équipé d'un système anticosmique.

\subsection{1. Étalonnage en rendement}

Des étalons aqueux, préparés dans la même géométrie de mesure et issus des solutions caractérisées par des 
mesures primaires, servent à définir la courbe de réponse en rendement du détecteur. Puisque la masse volumique de la matrice marquée $\left(0,37 \mathrm{~kg} \cdot \mathrm{L}^{-1}\right)$ est bien plus faible que celle des étalons aqueux usuels (environ $1 \mathrm{~kg} \cdot \mathrm{L}^{-1}$ ), il est nécessaire d'appliquer un facteur correctif de l'autoatténuation adapté afin de prendre en compte ces différences de masse volumique et de composition. Cette étape complexe et fastidieuse, décrite ci-après, pourrait être évitée si les laboratoires pouvaient directement utiliser ce type de matrices marquées pour étalonner leurs détecteurs.

\subsubsection{Correction d'auto-atténuation}

Les échantillons environnementaux ont des masses volumiques variables entre $0,1 \mathrm{~kg} \cdot \mathrm{L}^{-1}$ et près de $2 \mathrm{~kg} \cdot \mathrm{L}^{-1}$. Les coefficients d'atténuation massiques diffèrent d'un échantillon à l'autre principalement du fait de leur masse volumique plutôt que de leur composition chimique. Le facteur de correction d'auto-atténuation est obtenu par le rapport de l'atténuation dans les deux matrices considérées selon l'équation (1) :

$$
C_{\text {corr }}=\frac{\frac{\mu_{\text {Sol }}(E)}{\rho_{\text {Sol }}}}{\frac{\mu_{\text {Herbe }}(E)}{\rho_{\text {Herbe }}}} \cdot \frac{1-e^{-\frac{\mu_{\text {Herbe }}(E)}{\rho_{\text {Herbe }}} \cdot \rho_{\text {Herbe }} \cdot x_{\text {Herbe }}}}{1-e^{-\frac{\mu_{\text {Sol }}(E)}{\rho_{\text {Sol }}} \cdot \rho_{\text {Sol }} \cdot x_{\text {Sol }}}}
$$

où le coefficient d'atténuation linéique $\left(\mathrm{en}^{-1}\right)$ en fonction de l'énergie $E$ est noté $\mu(E), \rho$ est la masse volumique (en $\left.\mathrm{g} \cdot \mathrm{cm}^{-3}\right)$ et $x$ l'épaisseur $(\mathrm{en} \mathrm{cm}$ ) de matériau traversée. Les indices «Herbe» et «Sol» se réfèrent respectivement à la matrice marquée et aux solutions étalons. Le coefficient $\mu_{\text {Sol }}(E)$ est obtenu à partir de la base de données XCOM du NIST [12] pour l'acide chlorhydrique, milieu des étalons aqueux. Le coefficient $\mu_{\text {Herbe }}(E)$ est estimé à partir de mesures de la transmission (loi de Beer-Lambert) à travers un conteneur vide puis d'un conteneur rempli de matrice herbe non marquée de hauteur connue, de faisceaux de photons gamma collimatés produits par des sources radioactives ponctuelles $\left({ }^{152} \mathrm{Eu}\right.$ et ${ }^{133} \mathrm{Ba}$, entre $53 \mathrm{keV}$ et $\left.1,41 \mathrm{MeV}\right)$. La valeur de $\mu_{\text {Herbe }}(E)$ est alors obtenue en appliquant la relation (2) :

$$
\mu_{\text {Herbe }}(E)=\frac{1}{x_{\text {Herbe }}} \ln \frac{I_{0}(E)}{I(E)}
$$

où $I_{0}$ est l'intensité du faisceau parallèle des photons transmis à travers le conteneur vide et $I$ celle des photons transmis à travers le conteneur rempli avec une hauteur $x$ d'herbe non marquée. Ces mesures ont permis d'obtenir une courbe correspondant au facteur correctif des différences d'auto-atténuation en fonction de l'énergie des photons qui traversent la matrice marquée. Cette courbe, illustrée en figure 3, est décrite par une équation logarithmique du quatrième degré et est intégrée (ainsi que les incertitudes associées à sa détermination) dans le logiciel de traitement des données Interwinner 5.0 [13] afin d'en déduire l'activité massique des radionucléides.

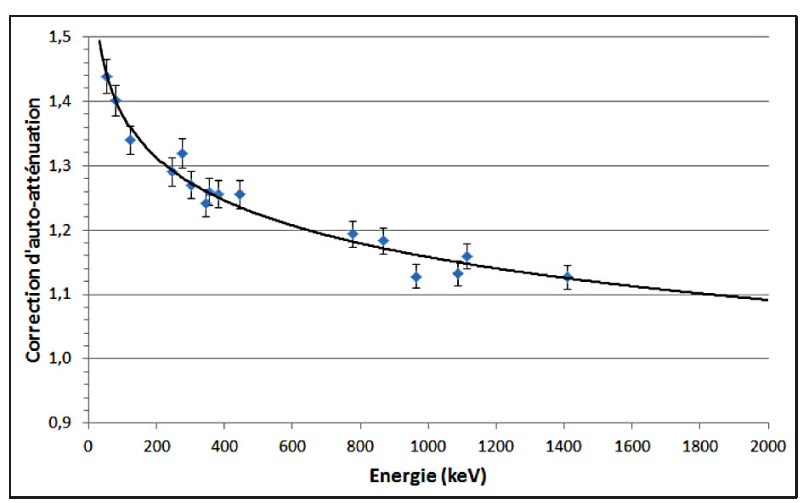

Fig. 3. - Courbe de correction des différences d'autoatténuation entre la matrice marquée et les étalons aqueux. Les barres d'incertitude sont données à $k=1$.

Ce facteur de correction est important, en particulier à basse énergie en dessous de $500 \mathrm{keV}$, et finit par tendre vers l'unité à haute énergie.

\section{5. Étude de l'homogénéité de la matrice produite}

Après les étapes de marquage et d'homogénéisation, nous avons décidé de tester l'homogénéité de la matrice produite à une échelle inférieure à celle des échantillons de 0,5 L. Des échantillons sont donc ouverts et la matrice est répartie dans dix conteneurs dix fois plus petits.

Ces sous-échantillons sont mesurés pendant $48 \mathrm{~h}$ par spectrométrie gamma. Le but était de vérifier l'homogénéité entre chaque sous-échantillon, et non d'en déduire les activités massiques des radionucléides. Ainsi, après les corrections adéquates de décroissance et de normalisation par la masse de matrice dans chaque sousconditionnement, les incertitudes associées à ces mesures proviennent majoritairement des statistiques de comptage.

Le test d'homogénéité choisi est une analyse de variance unilatérale (ANOVA) définie dans le guide ISO 35:2006 [14]. L'analyse de la variance permet de tester l'influence d'une ou de plusieurs variables qualitatives sur une variable quantitative, à un risque donné (risque alpha de $5 \%$ ici). L'objectif est de tester si tous les souséchantillons proviennent d'une même population et donc s'ils sont compatibles.

Chacun des sous-échantillons est mesuré trois fois. Pour analyser les résultats de ces mesures, la première étape consiste à décomposer la variation totale des données en deux composantes, à savoir l'écart dû au facteur (le conditionnement) et l'écart résiduel. Il est alors possible d'écrire que la somme des carrés des écarts est une composition de la somme des carrés des écarts dus au facteur testé et celle issue des résidus. La seconde étape est la comparaison des deux estimateurs de la variance au moyen d'un test de Fisher. Pour cela, les sommes des carrés des écarts calculées précédemment sont normalisées par leur nombre de degrés de liberté respectifs. Enfin, le rapport de ces estimateurs de la variance est comparé à un seuil de rejet issu de la loi de Fisher et dépendant du 
Tableau 3

Comparaison des valeurs de référence d'activité massique de la matière sèche mesurées par spectrométrie gamma avec celles calculées à partir de l'activité massique de la solution de marquage utilisée.

\begin{tabular}{|c|c|c|c|c|}
\hline Radionucléide & $\begin{array}{c}A_{\mathbf{r} \text { mes. }} \\
\mathrm{Bq} \cdot \mathrm{kg}^{-1}\end{array}$ & $\begin{array}{c}\boldsymbol{u}_{\mathrm{r} \text { mes. }} \\
\mathrm{Bq} \cdot \mathrm{kg}^{-1}\end{array}$ & $\begin{array}{c}A_{\mathrm{r} \text { calc. }} \\
\mathrm{Bq} \cdot \mathrm{kg}^{-1}\end{array}$ & $\begin{array}{c}\boldsymbol{u}_{\mathrm{r} \text { calc. }} \\
\mathrm{Bq} \cdot \mathrm{kg}^{-1}\end{array}$ \\
\hline \multicolumn{5}{|c|}{ Mélange de marquage } \\
\hline${ }^{22} \mathrm{Na}$ & 165,0 & 3,9 & 169,8 & 1,2 \\
\hline${ }^{60} \mathrm{Co}$ & 176,6 & 4,4 & 175,3 & 1,1 \\
\hline${ }^{109} \mathrm{Cd}$ & 894 & 29 & 906 & 11 \\
\hline${ }^{134} \mathrm{Cs}$ & 113,3 & 3,0 & 115,2 & 1,3 \\
\hline${ }^{137} \mathrm{Cs}$ & 171,9 & 4,3 & 174,3 & 1,3 \\
\hline \multicolumn{5}{|c|}{ Naturellement présent } \\
\hline${ }^{40} K$ & 1213 & 33 & I & 1 \\
\hline
\end{tabular}

risque alpha choisi et des deux degrés de liberté précédemment calculés. Si le rapport calculé reste inférieur à ce seuil, alors on en conclut qu'il n'existe pas de différence statistiquement significative entre les deux estimateurs de la variance. Ainsi, on considère que les dix populations sont «identiques » et que le facteur de variabilité testé (le sous-conditionnement ici) ne modifie pas l'activité initiale.

Pour tous les radionucléides testés, l'hypothèse nulle, supposant que les mesures sont indépendantes de l'échantillonnage, ne peut être rejetée au niveau seuil de risque choisi, elle est donc considérée comme vraie. La matrice est considérée comme homogène sur des souséchantillons de l'ordre de $50 \mathrm{~mL}$ ou bien $14 \mathrm{~g}$ soit un dixième du volume du conditionnement expédié pour le test interlaboratoire. En pratique, les écarts types des mesures réalisées sur les sous-échantillons de $14 \mathrm{~g}$ sont compris entre $2,1 \%$ pour le ${ }^{60} \mathrm{Co}$ et $3,0 \%$ pour le ${ }^{137} \mathrm{Cs}$. La dispersion observée pour le ${ }^{40} \mathrm{~K}$, naturellement présent, est de 2,5\%. Ces écart types sont faibles mais plus importants que la dispersion observée à plus grande échelle sur les échantillons de $0,5 \mathrm{~L}$ envoyés (entre $0,6 \%$ pour le ${ }^{22} \mathrm{Na}$ et $1,3 \%$ pour le ${ }^{134} \mathrm{Cs}, 1,1 \%$ pour le $\left.{ }^{40} \mathrm{~K}\right)$.

\subsection{Valeurs de référence et incertitudes associées}

Pour chacun des radionucléides, les valeurs de référence sont déterminées par spectrométrie gamma $\left(A_{\mathrm{r} \text { mes. }}\right)$ et calculées à partir des quantités de solution radioactives traçables utilisées $\left(A_{\mathrm{r} \text { calc. }}\right)$. Les incertitudes associées à ces valeurs de références sont mentionnées (respectivement $u_{\mathrm{r} \text { mes. }}$ et $u_{\mathrm{r} \text { calc. }}$ ) à $k=1$. Les rendements de marquage peuvent être évalués par le rapport des valeurs de référence mesurées et calculées (tab. 3 et 4).

Il convient de souligner que dans le cas de l'utilisation des rendements de marquage évalués ici pour le calcul des valeurs de référence de matrices marquées similaires, l'incertitude sur le rendement de marquage serait la contribution principale au bilan des incertitudes.

Sept échantillons de 0,5 L sur les vingt préparés sont mesurés par spectrométrie gamma pendant $72 \mathrm{~h}$ chacun
Tableau 4

Estimation des rendements de marquage à partir du rapport de l'activité de référence mesurée par spectrométrie gamma par l'activité de référence calculée via la masse de solution radioactive utilisée.

\begin{tabular}{|c|c|c|}
\hline Radionucléide & $\begin{array}{c}\text { Rendement } \\
(\boldsymbol{\%})\end{array}$ & $\begin{array}{c}\text { Incertitude }(\boldsymbol{k}=1) \\
\text { sur le rendement }(\boldsymbol{\%})\end{array}$ \\
\hline${ }^{2 \mathbf{2 2}} \mathbf{N a}$ & 97,2 & 2,5 \\
\hline${ }^{\mathbf{6 0}} \mathbf{C o}$ & 100,7 & 2,6 \\
\hline${ }^{109} \mathbf{C d}$ & 98,6 & 3,4 \\
\hline${ }^{\mathbf{1 3 4}} \mathbf{C s}$ & 98,4 & 2,8 \\
\hline${ }^{137} \mathbf{C s}$ & 98,6 & 2,6 \\
\hline
\end{tabular}

Tableau 5

Bilan d'incertitude de mesure $(k=1)$ associé aux valeurs de référence de la matrice végétale marquée pour le cas du cadmium 109.

\begin{tabular}{|c|c|c|}
\hline $\begin{array}{l}\text { Source } \\
\text { d'incertitude }\end{array}$ & $u_{\text {rel }}(\%)$ & Remarques \\
\hline Comptage & $0,8 \%$ & $\begin{array}{c}<0,5 \% \text { pour les autres } \\
\text { radionucléides }\end{array}$ \\
\hline Rendement & $2,7 \%$ & $\begin{array}{c}<1,5 \% \text { pour les autres } \\
\text { radionucléides }\end{array}$ \\
\hline $\begin{array}{l}\text { Auto- } \\
\text { atténuation }\end{array}$ & $1,7 \%$ & Quelle que soit l'énergie \\
\hline Homogénéité & $1,2 \%$ & $\begin{array}{c}<1,3 \% \text { dans tous les cas, } \\
1,1 \% \text { pour le }{ }^{40} \mathrm{~K} \text { (présent } \\
\text { naturellement) }\end{array}$ \\
\hline $\begin{array}{l}\text { Taux } \\
\text { d'humidité }\end{array}$ & $0,02 \%$ & / \\
\hline Pesée & $0,02 \%$ & l \\
\hline Bilan & $3,5 \%$ & \\
\hline
\end{tabular}

afin d'établir les valeurs de référence pour cette matrice. L'incertitude associée à ces mesures est composée des incertitudes de comptage, de l'incertitude sur la courbe de rendement du détecteur, de l'incertitude sur la correction d'auto-atténuation et de la variabilité des mesures sur les sept échantillons mesurés. Le tableau 5 présente le bilan d'incertitude relatif à la valeur de référence du ${ }^{109} \mathrm{Cd}$, l'émetteur le plus délicat à mesurer. Les valeurs des incertitudes dans le cas des autres radionucléides font l'objet de remarques spécifiques.

Dans le but d'utiliser des matrices marquées comme matériaux de référence, il faut en complément déterminer l'incertitude associée à leur stabilité dans le temps à plus grande échelle. Aucune évolution n'est observée, aux incertitudes de mesure près, entre les mesures réalisées juste après le marquage et celles faites près d'une année plus tard. Le guide ISO 31:2000 [15] ne recommande pas de mentionner de période de validité sur les documents accompagnant les matériaux de référence. Toutefois, dans sa version en cours de révision, ce point va évoluer. Depuis 2013, l'Agence Internationale de l'Energie Atomique (AIEA) mentionne une durée d'utilisation de dix ans pour les matériaux similaires à ceux de cette étude [16]. Il est également mentionné que le suivi de la stabilité sur cette période est en cours 
et que les utilisateurs seront informés dans le cas où une évolution serait observée.

\section{Test interlaboratoire organisé à partir de la matrice végétale marquée}

En 2012, un test interlaboratoire portant sur la mesure de la matrice végétale marquée avec des radionucléides émetteurs gamma dont la préparation a été évoquée précédemment a été organisé. Chaque laboratoire participant au test devait identifier les radionucléides présents et mesurer leur activité massique, de quelques centaines de $\mathrm{Bq} \cdot \mathrm{kg}^{-1}$, pour une activité massique de l'ensemble de l'ordre de $3 \mathrm{kBq} \cdot \mathrm{kg}^{-1}$ (de matière séche). Ce test, qui s'est terminé en novembre 2012, a permis aux participants de se préparer pour le test réglementaire de l'IRSN du premier semestre 2013. Afin de ne pas fausser les calculs des paramètres qui vont suivre, un test d'identification des valeurs aberrantes est mis en place pour chacun des radionucléides. Le test retenu est le test de Grubbs, suggéré dans la norme NF ISO 5725-2 [17].

\subsection{Analyse des résultats des participants}

A la demande des participants, les règles et traitements statistiques appliqués sur les résultats des laboratoires sont similaires à ceux pratiqués lors des tests réglementaires organisés par l'IRSN.

Ces trois critères sont consultables dans la note disponible à l'adresse suivante http://www. mesure-radioactivite.fr/public/IMG/pdf/5_criteres_ techniques.pdf.

Afin que chaque laboratoire puisse aisément analyser son résultat, une classe de précision lui est affectée selon trois critères distincts. La règle retenue comporte trois classes : « Satisfaisant », « Discutable », « Non Satisfaisant », par ordre de mérite décroissant.

Les trois critères de compatibilité sont les suivants : l'écart relatif, l'écart normalisé ainsi que le score-z. Dans le cas de ce test préliminaire regroupant moins de 10 participants, seuls les deux premiers critères ont été utilisés car le score-z n'aurait pas été pertinent. En effet, l'écart type interlaboratoire des résultats intervient au dénominateur dans le calcul de ce critère et il est jugé peu significatif en deçà de 10 participants.

\subsection{1. Écart relatif}

L'écart relatif $\left|e_{p, i}\right|$ est calculé selon :

$$
\left|e_{p, i}\right|=100 \times \frac{\left|A_{p, i}-A_{r, i}\right|}{A_{r, i}}
$$

où $\left|A_{p, i}-A_{r, i}\right|$ est la valeur absolue de l'écart constaté entre la valeur déterminée par le participant $p$ pour le $i^{\mathrm{e}}$ constituant identifié, $A_{p, i}$, et la valeur de référence $A_{r, i}$ de ce $i^{\mathrm{e}}$ constituant mesurée par le LNE-LNHB.
Tableau 6

Valeurs limites des deux critères présentés.

\begin{tabular}{|c|c|c|c|}
\hline Classification & Satisfaisant & Discutable & $\begin{array}{c}\text { Non } \\
\text { satisfaisant }\end{array}$ \\
\hline Écart relatif & $<15 \%$ & $15 \%-20 \%$ & $>20 \%$ \\
\hline $\begin{array}{c}\text { Écart } \\
\text { normalisé }\end{array}$ & $\leq 1$ & $1-1,3$ & $\geq 1,3$ \\
\hline
\end{tabular}

\subsection{2. Écart normalisé}

Le critère de compatibilité, prend en compte les deux couples de valeurs $\left(A_{p, i}, U_{p, i}\right)$ et $\left(A_{r, i}, U_{r, i}\right)$ où $U_{p, i}$ et $U_{p, i}$ sont les valeurs des incertitudes élargies $(k=2)$ associées respectivement à $A_{p, i}$ et $A_{r, i}$. Ce critère constitue une bonne indication de la maîtrise du processus de mesure qui ne peut être considéré comme totalement acquis que si le laboratoire a une vue complète et quantitative des causes d'incertitudes.

Le critère $\left|E n_{p, i}\right|$ est donc calculé selon :

$$
\left|E n_{p, i}\right|=\frac{\left|A_{r, i}-A_{p, i}\right|}{\sqrt{\left(U_{p, i}^{2}+U_{r, i}^{2}\right)}} .
$$

Afin d'éviter qu'une surestimation des incertitudes ne conduise à aider à obtenir un résultat satisfaisant, l'incertitude $U_{p, i}$ est limitée à $U_{p, i}^{*}=2 \sigma_{\mathrm{lab}, i}$ lorsque l'incertitude $U_{p, i}$ excède deux fois l'écart type de la distribution des résultats de l'ensemble des laboratoires pour ce même radionucléide $i: \sigma_{\mathrm{lab}, i}$.

\subsubsection{Classes de précision obtenues}

Le tableau 6 rassemble les valeurs limites des deux critères présentés pour obtenir les classes de précision « Satisfaisante », «Discutable » ou « Non Satisfaisante ».

\subsubsection{Remarque par rapport à l'application de la norme ISO 13528:2005}

La classification des résultats telle que proposée ici pour l'écart relatif et l'écart normalisé est spécifique des tests réglementaires français et se trouve être quelque peu différente de la classification qui découlerait de l'application de la norme ISO 13528:2005 [18] à partir des valeurs des scores $z$ suggérés (entre 2 et 3 ). Toutefois, ce point est à relativiser dans la mesure où les critères tels qu'appliqués ici se trouvent être légèrement durcis car le domaine de validité de la classe «Discutable» (pour l'écart relatif et l'écart normalisé) est moins large que celui préconisé par la norme ISO 13528:2005. De la même manière, la classe « Non Satisfaisant» est obtenue pour des valeurs plus faibles de ces critères.

\subsection{Résultats des participants}

Les résultats des participants sont uniformément répartis de part et d'autre des valeurs de référence car 
la moyenne des vingt-trois écarts relatifs obtenus est de $0,8 \%$. Sachant que la moyenne des incertitudes élargies mentionnées par les participants pour ces mesures est de l'ordre de $10 \%$, les résultats soumis sont tout à fait en accord avec les valeurs de référence.

Si nous nous intéressons aux résultats obtenus, radionucléide par radionucléide, l'isotope le mieux mesuré est le ${ }^{22} \mathrm{Na}$ pour lequel la valeur moyenne des écarts relatifs obtenus est de $-0,6 \%$ et le «moins bien mesuré » est le ${ }^{137} \mathrm{Cs}$ (avec une moyenne des écarts relatifs obtenus de $+2,7 \%$ ). Toutefois, c'est bien pour le ${ }^{109} \mathrm{Cd}$ que l'écart type sur l'ensemble des résultats soumis est le plus fort $(8,5 \%)$ comme attendu du fait de la faible énergie du photon gamma émis ( $88 \mathrm{keV})$ et de sa faible intensité $(<5 \%)$. La dispersion la plus faible des résultats soumis est obtenue pour le ${ }^{40} \mathrm{~K}$ qui n'a pas fait l'objet d'un marquage, étant naturellement présent dans l'herbe initialement prélevée.

Quel que soit le radionucléide considéré, les résultats des participants sont tous satisfaisants : d'une part les écarts relatifs entre les résultats et les valeurs de référence sont inférieurs à $10 \%$, d'autre part, aux incertitudes élargies $(k=2)$ près, ces résultats sont tous compatibles avec les valeurs de référence. Ces résultats tendent à montrer que les laboratoires participants réalisent des mesures de bonne qualité et que la matrice marquée réalisée est adaptée à ces tests (homogénéité, etc.).

\section{Conclusions}

Depuis le début du projet, en janvier 2009, les activités du LNE-LNHB en matière de mesures environnementales (faibles activités) sur des matrices variées se sont considérablement développées. La préparation de matrices environnementales marquées a débuté en 2012. Plusieurs méthodes de marquage ont été testées (marquage en milieu humide ou sec) ainsi que différents moyens d'homogénéisation (agitation tridimensionnelle ou par rotation simple). In fine, la méthode retenue est le marquage en milieu humide, dans l'acétone, au moyen d'un évaporateur rotatif. Le processus de marquage à l'aide d'un évaporateur rotatif a permis d'obtenir des résultats très intéressants puisque les rendements de marquage sont proches de $100 \%$. Après vérification systématique, ce point ouvre la perspective d'une diminution du nombre de mesures nécessaire à l'établissement des valeurs de référence. Pour les faibles valeurs d'activité, les temps de comptage étant considérables, il s'agit d'une possibilité très intéressante.

Le LNE-LNHB souhaite poursuivre la réalisation de diverses matrices traçables, de masse volumique et de composition proches de celles des échantillons usuellement prélevés pour le suivi de la radioactivité environnementale, afin de permettre aux laboratoires d'étalonner leurs instruments de mesure ou de tester leur capacité de mesure par le biais de tests interlaboratoires [19].

\section{Perspectives}

L'utilisation de la méthode de marquage à plus grande échelle de fabrication pourrait permettre, in fine, de produire des quantités plus importantes de matériaux marqués. Avec l'aide des autres laboratoires nationaux de métrologie, les valeurs de référence de ces matériaux pourraient être confirmées et ainsi certifiées. Ces «matériaux de référence » viendraient ainsi compléter l'offre, pas si importante, des catalogues de l'IRMM, de l'AIEA ou du NIST (laboratoire national de métrologie des EtatsUnis). Ces institutions favorisent en effet l'utilisation de matériaux marqués prélevés sur des sites pollués et caractérisés par la suite après homogénéisation.

Dans la continuité de ces travaux, le LNE-LNHB propose, en collaboration avec ses homologues européens, des actions conjointes en lien avec l'amélioration de la traçabilité des mesures, à la fois environnementales et en soutien à l'industrie. Il s'est ainsi engagé dans le projet européen de métrologie EMRP MetroNORM qui a débuté en septembre 2013 pour une durée de trois ans. Son objectif est d'améliorer les méthodes de mesure de matériaux naturellement radioactifs afin de permettre aux industriels d'optimiser leur gestion (impact environnemental et radioprotection des travailleurs) tout en préservant le rendement économique.

\section{Références}

[1] Nour S., InN K. et FILlibEn J., Development of the NIST rocky flats soil standard, J. Radioanal. Nucl. Chem., 277, 2008, 161-168.

[2] ShaKhashiro A. et al., IAEA-447, A new certified reference material for environmental radioactivity measurements, Appl. Radiat. Isot., 70, 2012, 1632-1643.

[3] Meresova J. WätJen U. et Altzitzoglou T., Determination of natural and anthropogenic radionuclides in soil - results of an European Union comparison, Appl. Radiat. Isot., 70, 2012, 1836-1842.

[4] DE Felice P. et al., The quality control in the production of spiked reference materials for gamma-ray spectrometry, Appl. Radiat. Isot., 49 (9-11), 1998, 1421-1427.

[5] Harms A. et Gilligan C., Development of synthetic environmental radioactivity reference materials, Appl. Radiat. Isot., 70, 2012, 1940-1943.

[6] NF-EN-ISO/CEI-17025:2005. Exigences générales concernant la compétence des laboratoires d'étalonnages et d'essais.

[7] COMAR, http://www.comar.bam.de, Base consultée le 27 novembre 2014

[8] Shakhashiro A. et al., Matrix materials for proficiency testing: optimization of a procedure for spiking soil with gamma-emitting radionuclides, Anal. Bioanal. Chem., 387, 2007, 2509-2515.

[9] Sill C.W. et Hindman F.D., Preparation and testing of standard soils containing known quantities of radionuclides, Anal. Chem., 46(1), 1974, 113-118. 
[10] Olson D.G. et BernabeE R.P., Preparation and analysis of high-quality spiked soil standards, Health Phys., 54(4), 1988, 451-459.

[11] DE SANOIT J., Silica spiked with mixed $\gamma$-ray standards for use as environmental reference material, Radiochim. Acta, 65, 1994, 249-257.

[12] Berger M.J. et al., XCOM: Photon Cross Section Database (version 1.3,. National Institute of Standards and Technology, Gaithersburg, MD. Disponible à http:// physics.nist.gov/xcom. 2005.

[13] Interwinner. Software version 5.05.1044 from ORTEC. Disponible à http://www.interwinner.com.

Article reçu le 11 décembre 2014, version révisée reçue le 3 juillet 2015.
[14] Guide ISO-35:2006, Matériaux de référence - Principes généraux et statistiques pour la certification.

[15] Guide ISO-31:2000, Matériaux de référence - Contenu des certificats et étiquettes.

[16] IAEA-448, Reference sheet, certified reference material, radium-226 in soil from oil field (2013), disponible à : http://nucleus.iaea.org/rpst/ReferenceProducts/ ReferenceMaterials/Radionuclides/IAEA-448/index. htm.

[17] NF ISO 5725-2, 1994, Méthode de base pour la détermination de la répétabilité et de la reproductibilité d'une méthode de mesure normalisée.

[18] ISO 13528:2005, Méthodes statistiques utilisées dans les essais d'aptitude par comparaisons interlaboratoires.

[19] Lourenço V. et al., Preparation of spiked grass for use as an environmental radioactivity reference material, Appl. Radiat. Isot., 87, 2014, 456-460. 